
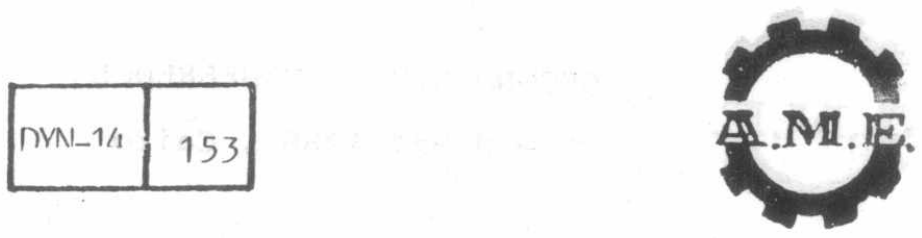

HILITARY TECHNICAL COLLEGE

CAIRO - EGYPT

\title{
VIBRATION ANALYSIS OF WANKEL ENGINE
}

\author{
* Dr. A.A.Omar, Eng. N.J.Yasin **
}

. ABSTRACT

- The studies for decreasing the generated vibrations of Internal Combustion - Engines are focused mainly on reciprocating engines, because of their wide. usage. But there are little studies interested in the vibrtations of rotary : W ankel engine.

The main objective of this research is focused to:

1. Develop a theoretical model of small NSU-Wankel engine for predicting the resultant shacking forces and moments.

2. Study the characteristics of the induced vibrations during operation.

Experimental investigation of the generated vibrations is used to assure the final results that are obtained from the theoretical analysis.

\section{INTRODUCTION}

Fleix Wankel presented his idea on rotary engines in 1954, designed his engine in 1958 as in Fig.l Engagement of stationary gear with internal gear control the movement of the rotor within its housing. The pitch diameters of the spur and internal gears are at the ratio $2: 3$, this constraint the motion of the rotor such that its angular velocity is one-third the crank speed.

\section{FORCE ANALYSIS}

Generallythe forces acting on the rotor, the crankshaft and housing are generated mainly from gas pressure in addition to $\wedge$ pex seals and eccentricities of rotor and crankshaft.

\section{Forces acting on the rotor}

The forces acting on the rotor during the operation of the engine are shown in Fig. 2. The rotor bearing reactions $\left(F_{b r x}, F_{b r y}, T_{r}\right)$ represent the resultant forces and torque due to gas forces $\mathrm{f}_{\mathrm{gr}}$, inertia or unbalance force $\mathrm{F}_{\mathrm{ir}}$, Apex

* Department of Design and Production Engineering

Faculty of Engineering, Ain Shams University, Cairo, Egypt

** Technical Institute, Karkuk, Iraq. 
seal forces $\mathrm{F}_{\text {ar }}$, gear forces $\mathrm{F}_{\mathrm{G}}$ and rotor assembly weight $\mathrm{W}_{\mathrm{r}}$

$F_{b r x}+F_{i x r}+F_{G t} \sin \Theta_{2}-F_{G n} \cos \Theta_{2}+\sum_{n=1}^{3} F_{g r n} \operatorname{Cos} \Theta_{f n}+\sum_{n=1}^{3} F_{a r n} \sin \alpha_{n}=0$

$F_{\text {bry }}+F_{i y r}-F_{G t} \cos \Theta_{2}-F_{G n} \sin \Theta_{2}+\sum_{n=1}^{3} F_{g r n} \sin \Theta_{f n}-\sum_{n=1}^{3} F_{a r n} \operatorname{Cos} \alpha_{n}-W_{r}=0$

- $T_{r}+T_{g r}-F_{i x r}\left[R_{2} \sin \Theta_{2}+\sqrt{E_{r}^{2}+S_{r}^{2}} \sin \left(\Theta_{2 / 3}+\tan ^{-1} S_{r} / E_{r}\right)\right]+\left(F_{i y r}-W_{r}\right)\left[R_{2} \cos \Theta_{2}+\right.$

$$
\left.\sqrt{E_{r}^{2}+S_{r}^{2}} \cos \left(\Theta_{2 / 3}+\tan ^{-1} S_{r} / E_{r}\right)\right]-F_{G t} R_{3}^{-T} a r=0
$$

where

$$
\Theta_{\mathrm{fn}}=\Theta_{2 / 3}+\frac{2 \pi}{3}(\mathrm{n}-1)
$$

\section{Forces on the crankshaft}

The main bearing reactions ( $\left.F_{b c x}, F_{p c y}, T_{0}\right)$ indicates the resultant forces and moment around crank center of fotation due to forces and moment from rotor, crankshaft inertia force $\mathrm{f}_{\mathrm{ic}}$ and the weight of the crannkshaft $\mathrm{W}_{\mathrm{c}}$

$$
\begin{aligned}
& F_{b c x}+F_{b r x}+F_{i x c}+F_{i x b^{*}}=0 \\
& F_{b c y}+F_{b r y}+F_{i y c}+F_{i y b^{*}}-W_{C}=0 \\
& T_{o}+T_{r}-\left(F_{i x c}+F_{i x b^{*}}\right) \sqrt{E_{C}^{2}+S_{C}^{2}} \cdot \sin \left(\Theta_{2}+\tan ^{-1} S_{c} / E_{C}\right) \\
& \quad+\left(F_{i y c}+F_{i y b^{*}}-W_{C}\right) \sqrt{E_{C}^{2}+S_{C}^{2}} \cdot \cos \left(\Theta_{2}+\tan ^{-1} S_{C} / E_{C}\right)=0
\end{aligned}
$$

where $\mathrm{F}_{\mathrm{ixb}^{*}}$, $\mathrm{F}_{\text {iyb* }}$ represent the additional inertia force due to the balancing weight that used to blance the eccentricity $\mathrm{R}_{2}$ of the center of rotor.

\section{Forces acting on the frame}

The resultant shaking forces and moments ( $\mathrm{F}_{\mathrm{fsx}}, \mathrm{F}_{\mathrm{fsy}}, \mathrm{T}_{\mathrm{fs}}$ ) which cause the frame to vibrate are genrated due to the forces and moments transmitted from crank, gas forces, gear forces affects in oposite direction to that obtained for the rotor and forces from apex seals

$$
\begin{gathered}
: F_{f s x}+\sum_{n=1}^{3} F_{g f n} \cos \Theta_{f n}-F_{G t} \sin \Theta_{2}+F_{G n} \cos \Theta_{2}+F_{b c x}+\sum_{n=1}^{3} F_{a f n} \cos \left(\alpha_{n}+\phi_{n}\right)=0 \\
F_{f s y}+\sum_{n=1}^{3} F_{g f n} \sin \Theta_{f n}+F_{G t} \cos \Theta_{2}+F_{G n} \sin \Theta_{2}+F_{b c y}+\sum_{n=1}^{3} F_{a f n} \sin \left(\alpha_{n}-\phi_{n}\right)=0 \\
T_{f s}+T_{g f}+F_{G t} \cdot R_{3}+T_{a f}+T_{o}=0
\end{gathered}
$$

The force $\mathrm{F}_{\text {ir }}$ is equal to the product of the gas pressure $\mathrm{P}_{\mathrm{n}}$ by the projected area $A_{p}$, which can be calculated approximately by simulating the rotor as an equal legs triangle, so

$$
A_{p r}=2 R_{4} \cdot t \cdot \sin \frac{\pi}{3}
$$


PYN-14 155

The total torque due to gas forces on the rotor can be dtermined as

$T_{g r}=\sum_{n=1}^{3} F_{x} \cdot R_{2} \sin \Theta_{2}-\sum_{n=1}^{3} F_{y} \cdot R_{2} \operatorname{Cos} \Theta_{2}=-\sum_{n=1}^{3} F_{g r n} \cdot R_{2} \sin \left(\Theta_{n}-\Theta_{2}\right)$

The force acting on the housing $E_{\text {gf }}$ can be obtained by multiplying the gas pressure inside the chambers by the projected area of the inner surface of the housing at each instant. The length of the inner surface $\mathrm{L}$ that belongs to each chamber can be defined by using the equations of the peritrochoid, then

$$
\mathrm{F}_{\mathrm{fgn}}=\mathrm{P}_{\mathrm{n}} \cdot \mathrm{L}_{\mathrm{n}} \cdot \mathrm{t}
$$

Determination of the volumetric change of the bombustion chamber will

help in getting the PV-diagram and instantaneous pressure as a funciton of crankshaft rotating angle. Volumetric change $A_{v}$ in. Wankel engines needs more complicated methods for its calculation: Generally, it can be described by using simple formulas, that have crankshaft rotating angle $\Theta_{2}$ as variable [1].

$$
\begin{aligned}
A_{v}= & \left(R_{2}^{2}+R_{3 / 4}^{2}\right) \pi-3 \sqrt{3 / 2} R_{2} R_{4} \sin \left(2 \Theta_{2 / 3}+\pi / 6\right)-\left(2 R_{4}^{2}+4 R_{2}^{2}\right) \cdot \pi / 6 \\
& +2 R_{2} R_{4} \sqrt{1-\left(3 R_{2} / R_{4}\right)^{2}}+\left(2 R_{4}^{2} / 9+4 R_{2}^{2}\right) \sin ^{-1}\left(3 R_{2} / R_{4}\right) .
\end{aligned}
$$

The analysis of the forces that operating on the apex seal is developed by kenji Matsuura, Kazuo Terasaki $[2,3]$. The inertia forces must be considered because of its large effect. after long operating time the inertia forces and their effect on the vibrations of the engine increases due to the increasing eccentricity. The eccentricity of the crankshaft is described by the notations $(E, S)$ along the $X$ and $\mathrm{Y}$ directions respectively. In addition to the assumed eccentricity $\left(E_{r}, S_{r}\right)$, the rotor is designed to have eccentricity $\mathrm{R}_{2}$ Fig.2.

$$
\begin{aligned}
& X=\sqrt{S_{C}^{2}+E_{C}^{2}} \cdot \operatorname{Cos}\left(\Theta_{2}+\tan ^{-1} S_{C} / E_{C}\right) \\
& Y=\sqrt{S_{C}^{2}+E_{C}^{2}} \cdot \sin \left(\Theta_{2}+\tan ^{-1} S_{C} / E_{C}\right)
\end{aligned}
$$

The analysis made at constant angular velocity $\ddot{\Theta}_{2}=0$, then

$$
\begin{gathered}
X=-\sqrt{S_{C}^{2}+E_{C}^{2}} \cdot \dot{\Theta}_{2}^{2} \cos \left(\Theta_{2}+\tan ^{-1} S_{C} / E_{C}\right) \\
Y=-\sqrt{S_{C}^{2}+E_{C}^{2}} \cdot \dot{\Theta}_{2}^{2} \sin \left(\Theta_{2}+\tan ^{-1} S_{C} / E_{C}\right) \\
F_{i x c}=-M_{C} X, F_{i y c}=-M_{C} \ddot{Y}
\end{gathered}
$$

Similarly, for rotor

$F_{i x r}=M_{r}\left[R_{2} \Theta_{2}^{2} \sin \Theta_{2}+\sqrt{E_{r}^{2}+S_{r}^{2}} \cdot\left(\dot{\Theta}_{2}^{2} / 9\right) \sin \left(\Theta_{2} / 3+\tan ^{-1} S_{r} / E_{r}\right)\right]$

$F_{\text {iyr }}=M_{r}\left[R_{2} \Theta_{2}^{2} \cos \Theta_{2} \sqrt{E_{r}^{2}+S_{r}^{2}} \cdot\left(\dot{\Theta}_{2}^{2} / 9\right) \sin \left(\Theta_{2} / 3+\tan ^{-1} S_{r} / E_{r}\right)\right]$

A balancing weight is added to the crankshaft to balance the unbalance force $\left(M_{r} \cdot B_{2}\right)$. But it suffers from the same assumed eccentricityof the crankshaft $\left(E, S_{C}\right)$ also. Thus after long operating time of engine, the interia forces that is generated from balancing weight are: 

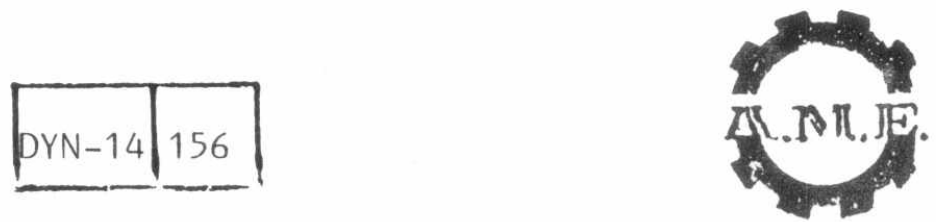

SECOND A.M.E. CONFERENCE

6 - 8 May 1986 , Cairo

$r$

$F_{\text {iyb }}=M_{r}\left[\left(\sqrt{E_{c}^{2}+S_{C}^{2}}-R_{2}\right) \cdot \Theta_{2}^{2} \sin \left(\Theta_{2}+\tan ^{-1} S_{C} / E_{C}\right)\right]$

\section{VIBRATION ANALYSIS}

The engine simulated as a rigid body with mass concentrated in the center of gravity and supported by four equivalent isolators. The equation of motion of the system can be written in matrix form using the same principles developed by Harry H. And Shelodn R. [4] as

$\left.\therefore \quad[M]\left(\ddot{q}_{i}\right)+[c]\left(\dot{q}_{i}\right)+[K] q_{i}\right)=\left(Q_{i}\right)$

where

[M] The matrix of mass and inertia,

$\left(q_{j}\right)$ Column matrix of motion of the center of mass in generalized coordinate,

[C] Square matrix for damping,

[K] Square matrix for stiffness,

$\left(Q_{i}\right)$ The column mtrix of forces in generalized directions.

The solution of this multidegrees system can be simplified by decoupling them using modal analysis [5]. The column matrix of displacement q. can be inncoupled as PY where $\mathrm{P}$ is the weighted modal matrix and $\mathrm{Y}$ is another column matrix. Substicute in eq. (21) and premultiply by $\mathrm{P}$, then

$$
\begin{aligned}
\mathrm{P}^{-\mathrm{T}} \mathrm{M} \overline{\mathrm{P}} \mathrm{Y}+\mathrm{P}^{-\mathrm{T}} \mathrm{C} \overline{\mathrm{P}} \dot{\mathrm{Y}}+\mathrm{P}^{-\mathrm{T}} \mathrm{K} \overline{\mathrm{P} Y} & =\mathrm{P}^{-\mathrm{T}} \mathrm{Q}_{\mathrm{i}} \\
\left.\mathrm{IY}_{\mathrm{i}}+2\right\} \mathrm{w}_{\mathrm{i}} \dot{\mathrm{Y}}_{\mathrm{i}}+\mathrm{w}_{\mathrm{i}}^{2} \mathrm{Y}_{\mathrm{i}} & =\mathrm{P}^{-\mathrm{T}} \mathrm{Q}_{\mathrm{i}}
\end{aligned}
$$

- where $I$ is a unit matrix, $W_{i}$ is the natural frequency in the coordinate i.Using

- Runge Kutta method and the uncoupled equations we can determine the system respone due to the applied excitation.

The effect of all components of the Wabkel engine aresuperimposed to produce complex functions of excitations that are acting on the system of isolators, beneath the base of engine. Thus the vibrations generated will be of complex nature. These complex vibrations can be resolved back into its basic frequency components using spectrum analysis method, which facilitate diagnosing the source of it among the various elements that construct the engine.

\section{EXPERIMENTAL INVESTIGATION}

: The equipments and instrumetns that are used in the experimental work are shown in Fig. 3. The records of spectrum components at different speeds and applied loads are obtained in logarithmic scale from the serial analyzer and in DB scale from real-time analyzer. Figs 4, 5 explain the variation in the spectrum components for two different speeds. The main components that declared in the figures are:-

a) Peaks at the fundamental frequency of the crankshaft ( $f_{d}$ ) and harmonics of it. It is shown also that the gear force, and the crankshaft inertia forces are functions of crankshaft rotting angle. Thus, these forces represent the main source of the above spectrum components.

b) Peaks at frequency $\left(f_{d} / 3\right)$, which is equal to the angular frequency of the rotor, and harmonics of it. 
c) Peaks at frequency $(2 \mathrm{fd} / 3)$ and harmonics of it. These spectrum generated due to the apex seal force component on the frame, and the moment that comes from the force between meshing gears. The peaks at $(\mathrm{fd})$ are less than peaks at $(\mathrm{fd} / 3),(2 \mathrm{fd} / 3)$ at speed $2000 \mathrm{RPM}$. This case is reversed at speed 6000 RPM. Fig. (6) shows the spectrograms for speed 4000 RPM. The peaks occurs at $(\mathrm{fd}),(\mathrm{fd} / 3)$ and its harmonics. There are other undefined peaks which may be due to the effect of bearings.

\section{Comparison between experimental and theoretical results}

The difference in peaks between the theoretical and experimental components - is in the range $4-46 \%$. It is seen that the error at the region behind ( $\mathrm{fd}$, - $f d / 3,2 f d / 3)$ is less than that at higher frequencies. This deviation may be due to
a) The intake and exhaust wave action,
b) The transmission errors of the gear system,
c) The generated peaks from the roller bearings.

\section{REFERENCES}

1. Kenichi Yamamoto, "Rotary engine", Toyo Kogyo Ltd,1971.

2. Kenji Matsuura, Kazuo Terasaki and Ichiro Watanabe, "IThe relative behaviour of a Rotory Engine Apex seal to the wall of a slot", bull. of the JSME, Vol. 19, No. 137, Nov. 1978.

3. Kenji Matsura, Kazuo Terasaki and Ichiro Watanabe, "The behaviour of a Rotory Engine. Apex seal against the trochoidal surface", Bull. of the JSME, Vol.21, No. 161,Nov. 1978.

- 4. Cyril M. Harris, Charles E. Crede,"Shock and Vibration Hand-book", Vol. 1, 1961.

5. William T. Thomson,"Vibration Theory and Appliction", 1969.

\section{NOMENCLATURE}

$\mathrm{n} \quad$ : The order number of the chamber,

$\mathrm{R}_{3}$ : Pitch circle radius of the rotor gear (internal gear),

$\mathrm{R}_{4}$ : Distance between center of rotor and center of gravity of apex seal.

$\mathrm{T}_{0} \quad$ : The generated torque on the crankshaft,

$: T_{\text {af }}:$ The generated torque from the apex affect on the frame,

$\mathrm{T}_{\text {ar }}$ : The generatged torque from the apex affect on the rotor,

$t \quad:$ The width of the rotor,

$\Theta_{\mathrm{fn}}$ : Effective angle of the force $F_{n}$ measured form $X$-axis, 


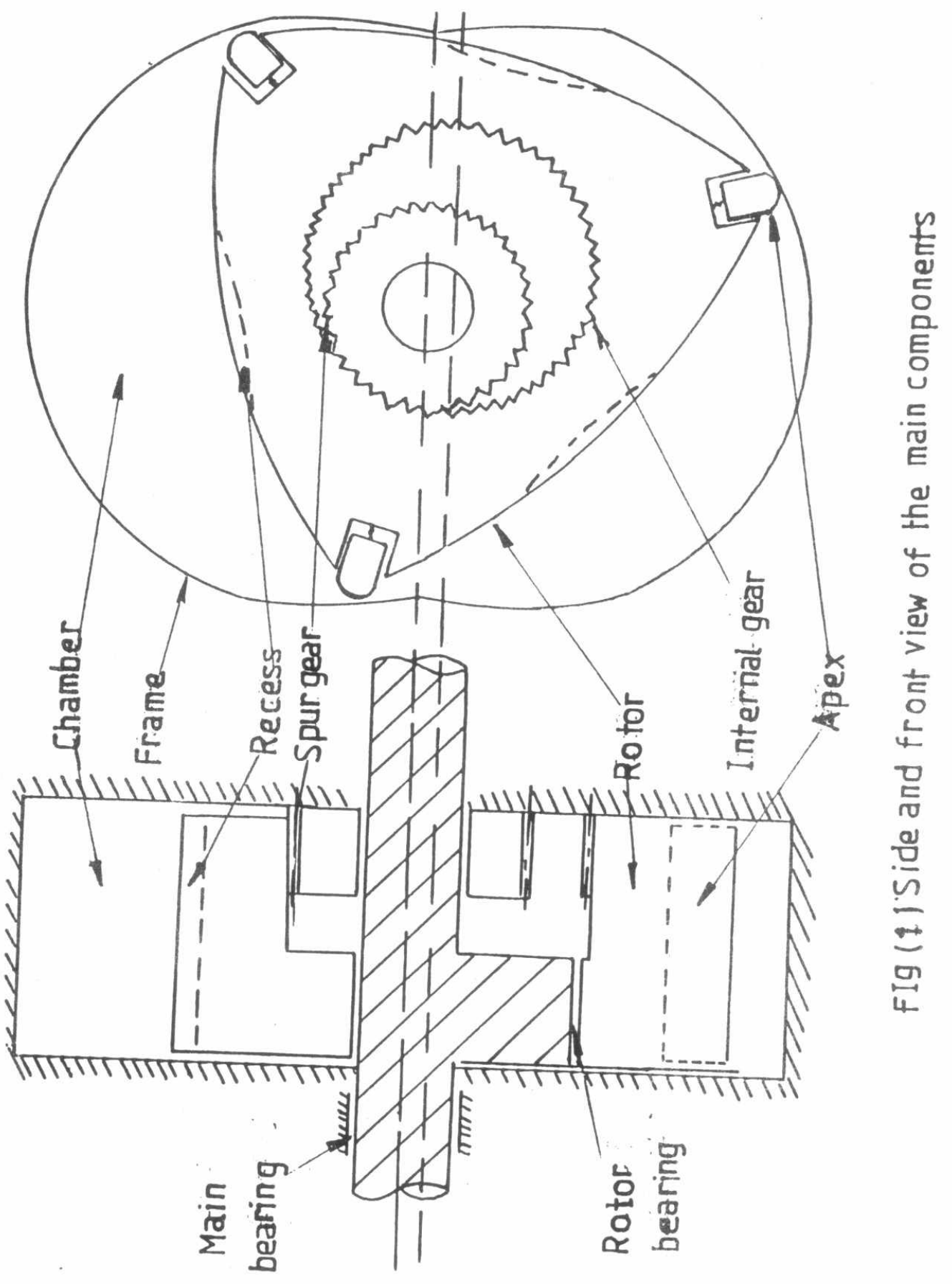




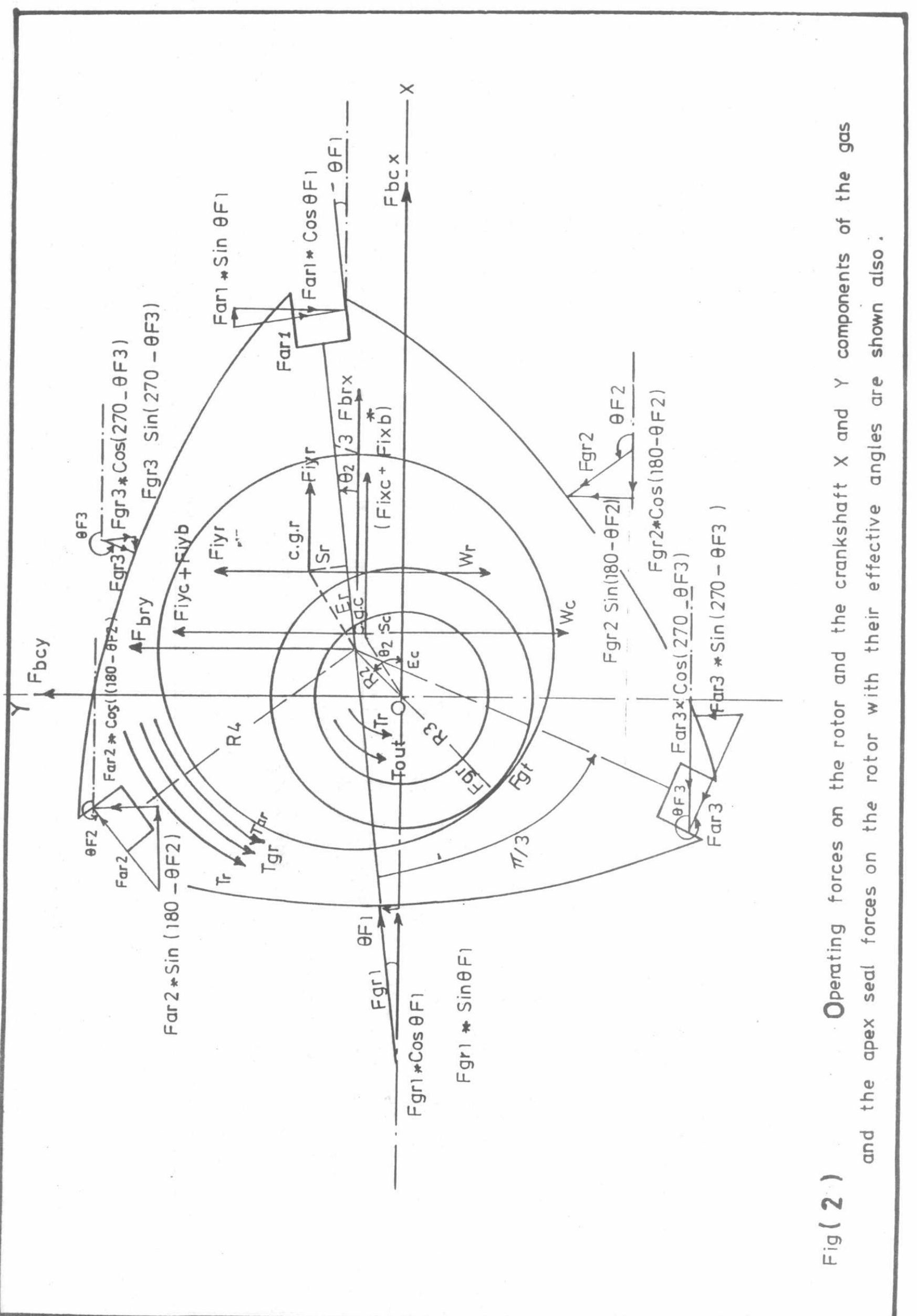




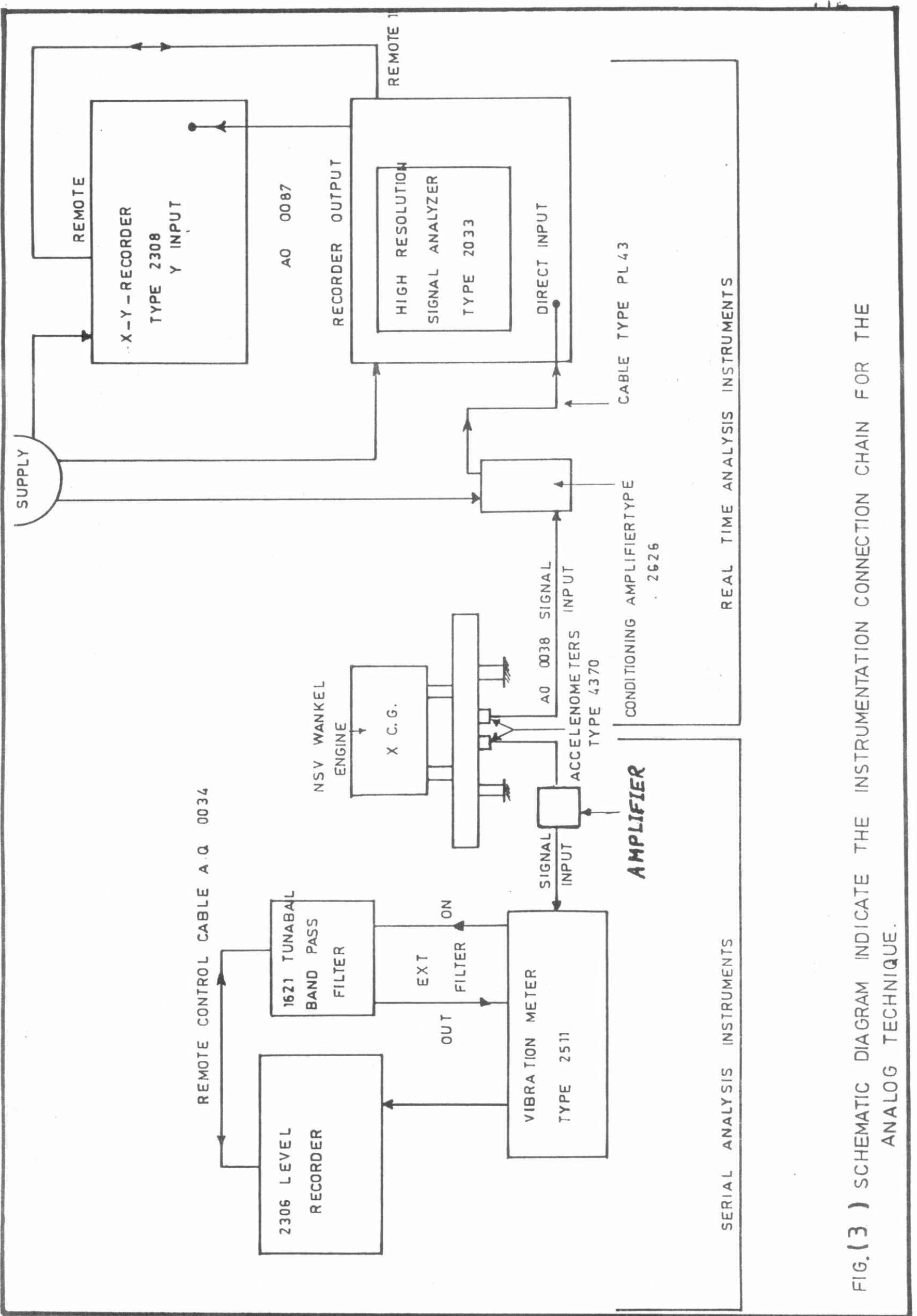




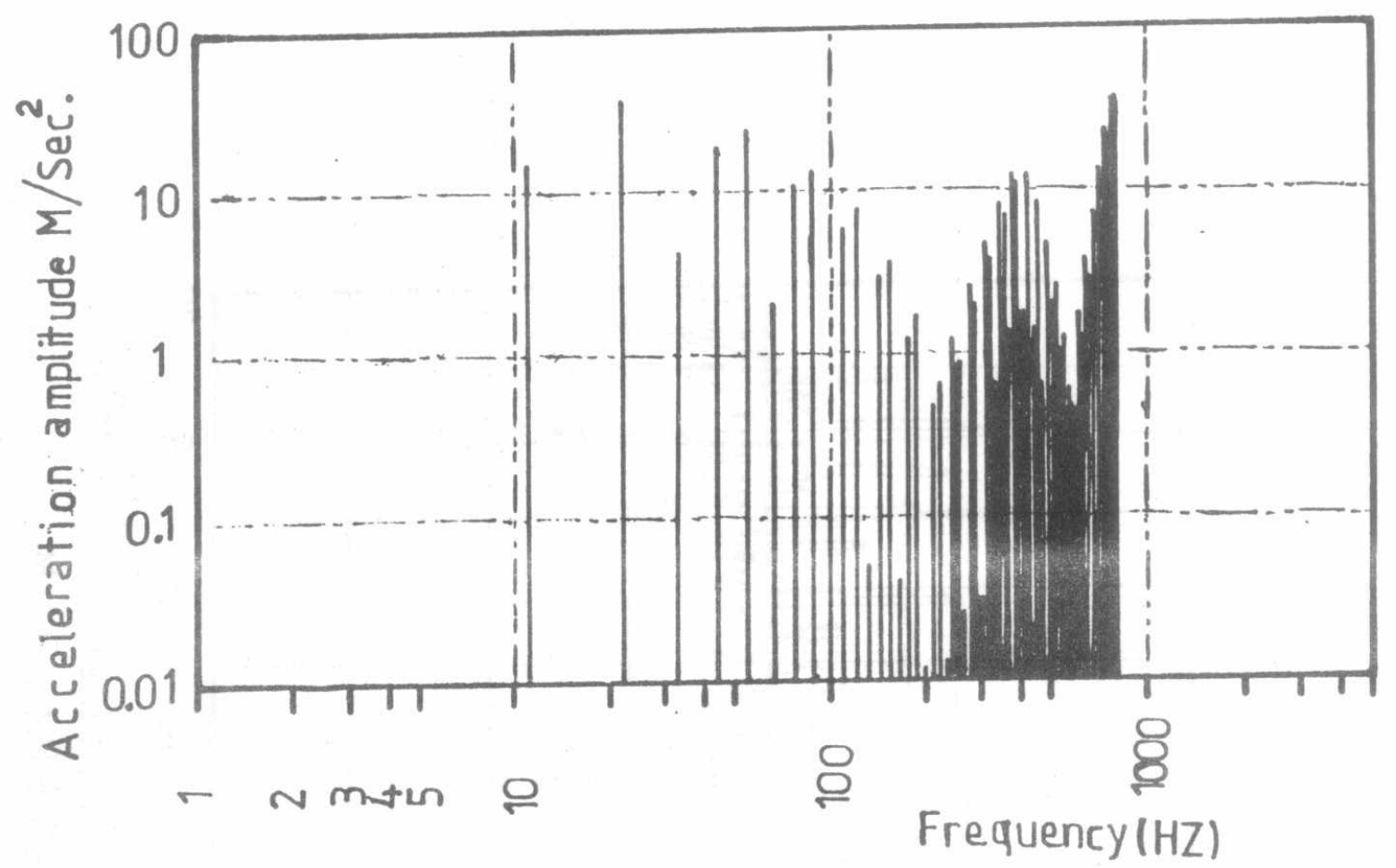

Fig.(4) The spectrum components at 2000 RPM.

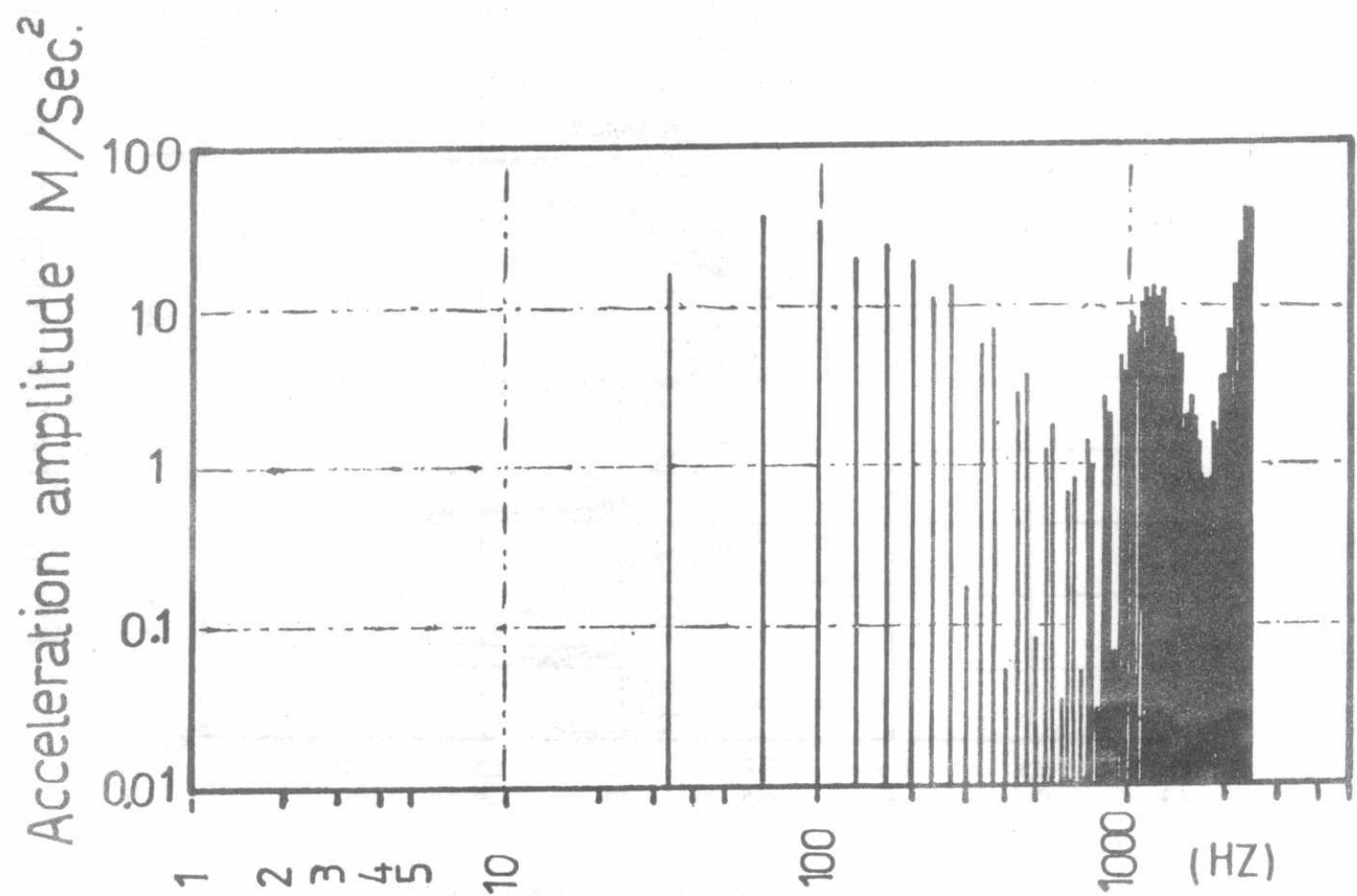

Fig. (5) The spectrum components at 6000 RPM. 


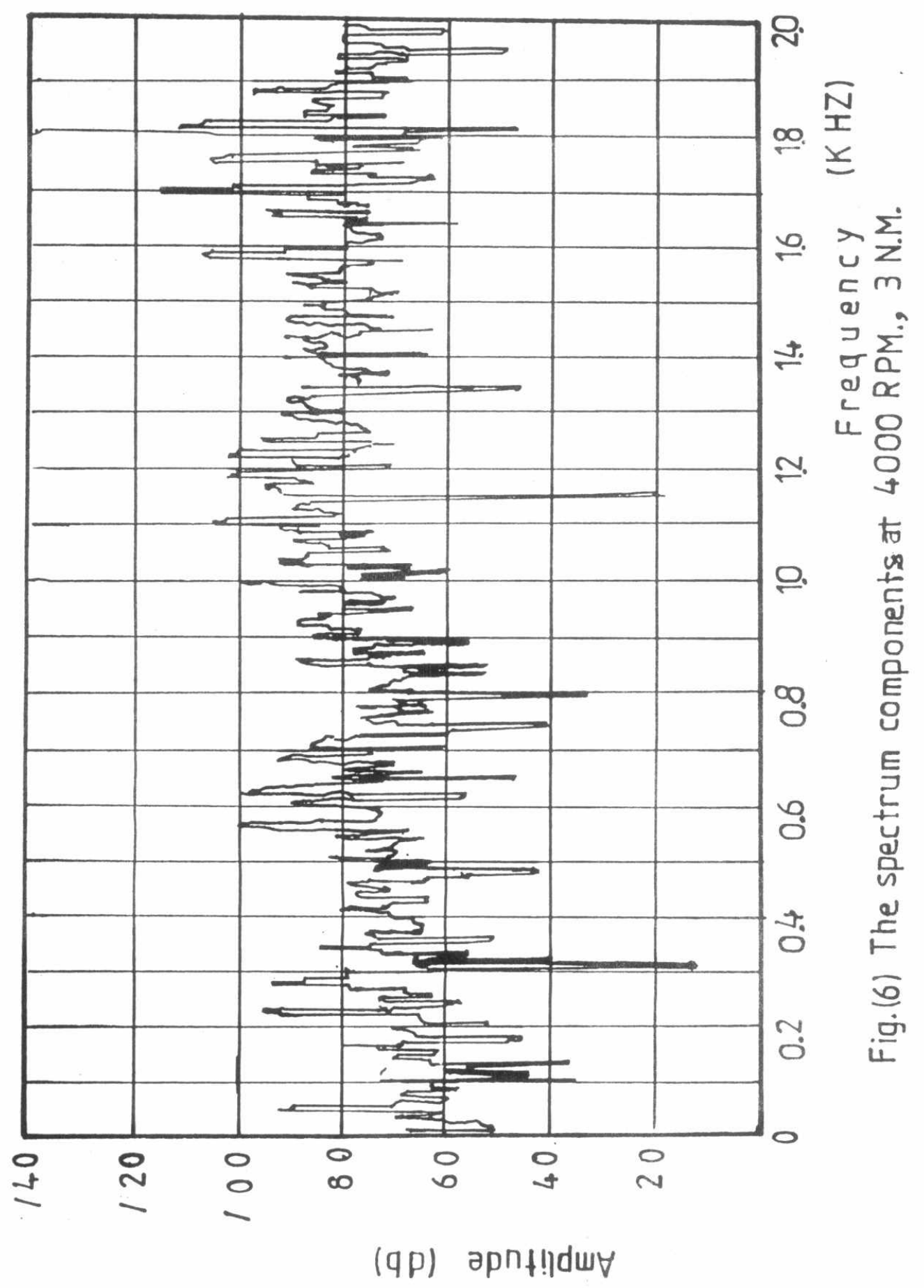

